\title{
Determinants, consequences and potential solutions to poor adherence to anti-osteoporosis treatment: results of an expert group meeting organized by the European Society for Clinical and Economic Aspects of Osteoporosis, Osteoarthritis and Musculoskeletal Diseases (ESCEO) and the International Osteoporosis Foundation (IOF)
}

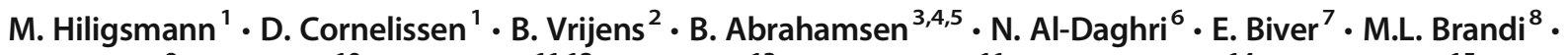

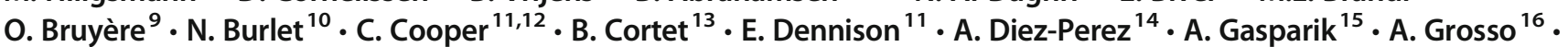 \\ P. Hadji ${ }^{17}$ - P. Halbout ${ }^{18}$ - J.A. Kanis ${ }^{19,20}$ • J.M. Kaufman ${ }^{21} \cdot$ A. Laslop ${ }^{22} \cdot$ S. Maggi ${ }^{23} \cdot$ R. Rizzoli $^{7} \cdot$ T. Thomas $^{24}$. \\ S. Tuzun ${ }^{25} \cdot$ M. Vlaskovska ${ }^{26} \cdot$ J.Y. Reginster ${ }^{6,9}$
}

Received: 13 June 2019 / Accepted: 18 July 2019 / Published online: 7 August 2019

(C) The Author(s) 2019

\begin{abstract}
Summary Many patients at increased risk of fractures do not take their medication appropriately, resulting in a substantial decrease in the benefits of drug therapy. Improving medication adherence is urgently needed but remains laborious, given the numerous and multidimensional reasons for non-adherence, suggesting the need for measurement-guided, multifactorial and individualized solutions. Introduction Poor adherence to medications is a major challenge in the treatment of osteoporosis. This paper aimed to provide an overview of the consequences, determinants and potential solutions to poor adherence and persistence to osteoporosis medication. Methods A working group was organized by the European Society on Clinical and Economic Aspects of Osteoporosis, Osteoarthritis and Musculoskeletal diseases (ESCEO) to review consequences, determinants and potential solutions to adherence and to make recommendations for practice and further research. A systematic literature review and a face-to-face experts meeting were undertaken. Results Medication non-adherence is associated with increased risk of fractures, leading to a substantial decrease in the clinical and economic benefits of drug therapy. Reasons for non-adherence are numerous and multidimensional for each patient, depending on the interplay of multiple factors, suggesting the need for multifactorial and individualized solutions. Few interventions have been shown to improve adherence or persistence to osteoporosis treatment. Promising actions include patient education with counselling, adherence monitoring with feedback and dose simplification including flexible dosing regimen. Recommendations for practice and further research were also provided. To adequately manage adherence, it is important to (1) understand the problem (initiation, implementation and/or persistence), (2) to measure adherence and (3) to identify the reason of non-adherence and fix it.

Conclusion These recommendations are intended for clinicians to manage adherence of their patients and to researchers and policy makers to design, facilitate and appropriately use adherence interventions.
\end{abstract}

Keywords Adherence $\cdot$ Determinants $\cdot$ Osteoporosis $\cdot$ Persistence $\cdot$ Solutions

Electronic supplementary material The online version of this article (https://doi.org/10.1007/s00198-019-05104-5) contains supplementary material, which is available to authorized users.

M. Hiligsmann

m.hiligsmann@maastrichtuniversity.nl

Extended author information available on the last page of the article

\section{Introduction}

Poor or non-adherence to medication remains a major problem in most chronic diseases, including osteoporosis. Effective and safe medications are available to reduce the risk of fractures [1], but numerous patients do not initiate treatment for osteoporosis or do not take it appropriately, resulting in a substantial clinical and economic burden [2]. Initiation of 
osteoporosis therapy is even poorer than in other disease areas, with a substantial decrease in bisphosphonates initiation observed in the last few years in Europe and the USA [3, 4]. Investing in medication adherence could improve health outcomes and health system efficiency [5].

Poor or non-adherence with osteoporosis medications is not a new problem. Several studies have already assessed the consequences and burden of non-adherence with osteoporosis medications being one of the major challenges of successful osteoporosis management [6] and have highlighted the urgency of managing medication adherence. In 2012, a systematic review of interventions to improve adherence to osteoporosis medications suggested few high-quality studies and mixed effects of interventions [7]. In recent years, osteoporosis management has evolved (e.g. new treatments, new diagnostic tools, FLS organizations), more interventions to improve adherence have been tested and a better understanding of the determinants of non-adherence is available.

Considering the burden of non-adherence with osteoporosis medications and the need to provide up-to-date recommendations to manage medication adherence, a working group was convened by the European Society on Clinical and Economic Aspects of Osteoporosis, Osteoarthritis and Musculoskeletal Diseases (ESCEO) to review the consequences and determinants of poor adherence and interventions to manage adherence and improve persistence to osteoporosis medication. In addition, this paper aims to provide recommendations for practice and for further research. Information included in this review could be of value to help not only clinicians to manage adherence of their patients but also for researchers and policy makers involved in osteoporosis.

\section{Methods}

An international working group was formed to review the consequences and determinants of poor adherence and interventions, to manage adherence and improve persistence to osteoporosis medication and to provide recommendations for practice and for further research. The working group comprised clinical scientists, researchers (including experts in adherence) and a patient selected by the Scientific Advisory Board of ESCEO.

In preparation for a working group meeting, a systematic literature review was conducted to identify articles about the determinants and interventions to improve adherence or persistence to osteoporosis medications. The literature search was conducted for articles up to December 2018 in PubMed. Further details about the search strategy and inclusion of articles can be found in Cornelissen et al. [8]

A face-to-face meeting then took place on 22 January 2019. The meeting started with three short presentations about the clinical and economic consequences of non-adherence to osteoporosis medications (JYR), the determinants of nonadherence to osteoporosis medications $(\mathrm{MH})$ and the potential solutions to improve adherence to anti-osteoporosis medications (DC). A group discussion led by MH and ADP followed. Each participant had the opportunity to comment on the determinants, consequences and potential solutions and then to provide recommendations for practice and further research. Following this meeting and a symposium that was held at the World Congress of Osteoporosis (WCO-IOF-ESCEO congress, 6 April 2019), members of the writing team (MH, DC, $\mathrm{BV}, \mathrm{BA}, \mathrm{JYR}, \mathrm{JK}, \mathrm{AG}, \mathrm{PH}$ ) reviewed a first version of the article drafted by $\mathrm{MH}$; this was then reviewed and commented on by all members of the working group.

\section{Terminology}

In the literature, a number of terms such as 'adherence', 'compliance', 'concordance' and 'persistence' have been used to define how patients take their medicines [9]. In 2012, a collaboration of European research groups in the field of medication adherence funded by the European Commission suggested the $\mathrm{ABC}$ taxonomy for describing and defining adherence to medications [9], which will be used in this paper. Adherence to medication is defined as 'the process by which patients take their medications as prescribed, composed of $\mathrm{A}$ ) initiation, B) implementation and C) discontinuation' $[9,10]$. Initiation occurs when the patient takes the first dose of a prescribed medication, discontinuation when the patient stops taking the medication and implementation is the extent to which a patient's actual dosing corresponds to the prescribed dosing regimen, from initiation until the last dose. Medication persistence is further defined as the length of time between initiation and the last dose, which immediately precedes discontinuation [9]. Overall, this definition is in agreement with the definition of the Group for the Respect of Ethics Excellence in Science in osteoporosis [11], with the exception that implementation has replaced compliance, and initiation is preferred over primary adherence.

\section{Adherence to osteoporosis medications, clinical and economic consequences}

In a recent review including 124 studies, the prevalence of medication adherence ranged from 12.9 to $95.4 \%$ [12]. Several studies have reported that initiation, implementation and persistence to osteoporosis medications are suboptimal. In line with treatment initiation rates in other diseases [13], about $20-30 \%$ of patients do not initiate a treatment after a prescription for oral bisphosphonates [14]. In addition, patients on bisphosphonates frequently miss doses and therefore do not implement as prescribed [15]. Persistence rates at 1 year for 
oral bisphosphonates are commonly estimated between 16 and $60 \%$ [16-18], in line with a review of 95 clinical studies from different disease areas suggesting that about $40 \%$ of patients discontinued at 1 year [19]. For example, in a large observational Belgian study, persistence rates to oral bisphosphonates were estimated at $39.5 \%$ at 1 year, without a gap of more of 5 weeks in treatment [20], while only $48 \%$ of patients were adherent (defined as a medical possession ratio $(\mathrm{MPR}) \geq 80 \%)$. In another study, van Boven et al. showed that persistence to weekly oral bisphosphonates was about $60 \%$ at 1 year and decreased to $25 \%$ at 5 years [21], in line with a Danish study [22]. Other studies have even reported that less than $20 \%$ were still on treatment as soon as after 12 months after treatment initiation [23]. Differences in methodologies (e.g. permissible gap to define persistence, adherence measures, incorporation of switchers) could explain the differences between studies and made direct comparison between them difficult.

Further, osteoporosis remains largely underdiagnosed and a substantial number of patients at increased risk of fractures do not even receive any prescription for osteoporotic therapy [24] or only calcium and vitamin D supplementation. The osteoporosis treatment gap was estimated between 25 and $95 \%$ in European countries [25]. Even in randomised controlled trials, persistence and adherence with therapy decline over time, and any reduced effectiveness caused by suboptimal adherence is to some extent already captured in clinical trials.

Different profiles of non-adherent patients could be identified [26]. Some patients never initiate a treatment, while others delay initiation of therapy. There are patients who frequently miss doses, and multiweek drug holiday periods have also been observed. Several patients further discontinue treatment earlier than prescribed. In a USA study, it was also observed that among discontinuers, about 45\% [27] reinitiated therapy, with the majority doing so within 6 months of discontinuation, suggesting that many patients are thus exposed to multiple episodes of starting and stopping of drug actions. Finally, several medication switchers have been reported [28]. Failure to take account of switchers could distort estimates of adherence and persistence.

Poor persistence and adherence to osteoporosis medications reduce the potential benefits of osteoporosis therapy, lowering gains in bone mineral density and resulting in increased risk of fragility fractures [29]. Two systematic reviews and meta-analyses assessed the impact of adherence/ persistence to oral bisphosphonates on fracture risk. The meta-analysis of Ross et al. [30] indicated that fracture risk increased by approximately $30 \%$ with non-adherence (defined as medical possession ratio $<80 \%$ ) and by 30 to $40 \%$ with non-persistence. Similarly, Imaz et al. [31] reported that low adherence to oral bisphosphonates was significantly associated with increased non-vertebral fracture risk (relative risk
(RR) of 1.16), increased hip fracture risk (RR 1.28) and increased vertebral fracture risk (RR 1.43).

Studies reporting adherence to osteoporosis medication and its relationship with fracture risk mainly focused on oral bisphosphonates, although data for other medications (e.g. teriparatide, raloxifene, denosumab or zoledronic acid) also revealed suboptimal levels $[32,33]$. In a large US study, persistence to teriparatide and denosumab at 1 year were estimated at $69 \%$ and $58 \%$ [34]. Non-persistence to denosumab could further lead to important health problems as discontinuation with denosumab has recently been shown to be associated with rapid bone loss and increased risk of multiple vertebral fractures [35]. Persistence to yearly intravenous injection of zoledronic acid is also not convincing, as suggested by a study that showed that only one-third of patients agreed to a second administration after 1 year [36]. Furthermore, for some osteoporosis medications, persistence to generic formulations has been shown to be poorer than for branded formulations [37, $38]$.

A few model-based studies have estimated the economic consequences of non-adherence at a population level [2, 39-42], suggesting important clinical and/or economic implications of poor adherence/persistence with osteoporosis medications. For example, poor adherence with osteoporosis medications resulted in about $50 \%$ reduction in the potential benefits observed in clinical trials (in terms of fractures prevented and quality-adjusted life-years (QALY)) and a substantial deterioration of the cost-effectiveness resulting from these medications [2, 40]. Medication adherence has further become an important factor for inclusion in cost-effectiveness analyses in osteoporosis [43-45]. Interestingly, economic studies have also suggested that interventions to improve adherence may likely confer cost-effectiveness benefits [43]. Improving adherence to medication could lead to greater benefits that designing a new more effective drug [46].

\section{Determinants of non-adherence}

Adherence is a complex multidimensional phenomenon determined by the interplay of several factors. Numerous determinants of non-adherence have been identified in the literature $[12,47]$. The World Health Organization has classified these factors into five main categories, i.e. patient-related, therapyrelated, condition-related, health system and socio-economic factors [5]. Recently, Yeam et al. [12] conducted a systematic literature review up to January 2018 to review and identify factors that influence patients' adherence to anti-osteoporotic therapy. A total of 24 factors and 139 subfactors were identified from 124 relevant studies. The authors presented types and number of studies that presented the case for and against each specific factor and revealed that patient-related factors 
were the most commonly studied category followed by therapy-related and condition-related category.

Condition-related factors that were associated with poorer medication adherence included polypharmacy and having gastro-intestinal diseases. History of falls, fractures and screening for osteoporosis were however not associated with higher adherence. This is important as patients with a recent fracture are at imminent risk of a further fracture [48]. Patientrelated factors associated with poorer medication adherence included male gender, lower education levels, misconceptions about osteoporosis and lack of perceived benefits of therapy, whereas higher age was associated with higher medication adherence but only in half of the studies. Among therapyrelated factors, medication side effects, complex instructions for medication administration and complex medication regimens were associated with poorer adherence and lower dosing frequency with higher adherence, while a history of antiosteoporosis treatment was not found to be a predictor of adherence. Health system-based factors associated with poorer medication adherence included care under different medical specialties and lack of patient education (information sharing, counselling from healthcare professional, etc.). Socio-economic-related factors associated with poorer medication adherence included current smoking, lower income level and lack of medical insurance coverage [12].

This review did not classify the factors according to adherence level, i.e. initiation, implementation and persistence. In another review of systematic reviews that covered 19 disease areas [47], a total of 771 individual factors of medication adherence were identified of which most were determinants of implementation, and only 47 determinants of persistence with medication and no determinant were specifically provided for initiation. In the field of osteoporosis, the patient perceived need for treatment, patient knowledge, bone density testing, improved patient-provider relationship, hospitalizations and prescription use have been shown to be positively associated with initiating osteoporotic therapy [49-52]. Reasons for not initiating osteoporotic therapy also include lack of motivation, concern about side effects and medication costs [52]. Reasons for poor implementation and persistence with osteoporosis therapy include side effects of treatment, difficulties with medication intake (e.g. $30 \mathrm{~min}$ before breakfast), inconvenient dosing regimen, concern about treatment, no perceived benefits, drug cost, misinformation, insufficient motivation or dissatisfaction with their doctor visits $[47$, 53-55]. Adherence to osteoporosis medications is simply a matter of patient choice, and is due mostly to deliberative choice [56]. However, reasons for non-adherence can also be unintentional, resulting from forgetfulness, especially in holiday period [53], and some irrational behaviour could also be reported. Factors involved in treatment initiation may further differ than factors involved in persistence or implementation. As example, polypharmacy has been shown to have a positive effect on treatment initiation with patients who have not previously taken medication being less likely to start [14] while persistence and implementation are generally lower in patients on multiple medications [12]. Lau et al. conducted focus groups in Canada to understand reasons for non-adherence and included different patients' quotes regarding belief in the importance of taking medications for osteoporosis, medication-specific factors, beliefs regarding medications and health, relationships with healthcare providers and information exchange [57].

The patient participating at our working group stressed the importance of giving a positive message to the patient and not exaggerating the consequences if the patient does not take the drug. Web and social media nowadays also attract a lot of attention and could further negatively influence adherence to treatment.

Reasons for non-adherence are therefore numerous and multidimensional for the same patient. Each patient's reason(s) for non-adherence is different, depending on the interplay of these multiple factors, and could also change over time and be different for each key element of adherence. Medication adherence is therefore not predictable, suggesting the need for individualized solutions.

\section{Review of interventions}

In 2012, Hiligsmann et al. [7] reviewed and critically appraised interventions to improve adherence and persistence to osteoporosis medications. A total of 20 studies tested a patient adherence intervention and reported quantitative results on adherence. Education programs (e.g. written materials, counselling, motivational interviewing, combination) were the most frequent intervention $(n=9)$. Although patient education improved medication adherence in four studies, two large-scale randomized studies reported no benefits [58, 59]. Monitoring/ supervision by bone mineral density or bone turnover markers were not shown to be associated with improved adherence. However, a positive message revealing a good bone turnover marker response was associated with a significant improvement in persistence [60]. Simplification of dosing regimens (with and without patient support program), electronic prescription and a pharmacist intervention was associated with improved adherence but only in couple of studies.

For the purpose of this working group, the previous systematic review was updated. A description of the literature search, data and critical appraisal can be found in the article of Cornelissen et al. [8]. A total of 15 studies were identified between June 2012 and December 2018. Very few highquality studies (such as RCTs) were identified, and interventions were mainly single component intervention. Interventions were classified as patient education and/or support $(n=9)$, monitoring and supervision $(n=2)$, flexible 
dosing regimen and patient support $(n=3)$ and interdisciplinary collaboration $(n=1)$. In each subtype of interventions, mixed results on adherence/persistence were found where some studies were shown to be successful and others not (see next section). Interventions which include counselling with education and/or flexible dosing regimens seem to be most effective, as well as interventions aiming on testing and initiating medication.

\section{Recommendations for practice}

Many factors affect medication adherence. Single interventions could work but certainly not for all patients. There is not one single intervention to manage adherence. Some recommendations to improve adherence are made below.

It is of primary importance to emphasize the need for treatment adherence and to talk about adherence with patients. To adequately manage medication adherence, at least three stages are recommended:

1. What is the adherence problem (initiation, implementation and/or persistence)? It is key to identify the element(s) of non-adherence problem(s) that you want to address with the patient(s).

2. How to measure adherence? Different methods are available to measure the elements of medication adherence. Prescription refills and databases are appropriate methods to measure initiation and discontinuation of therapy [61] while electronic monitoring is the preferred method for implementation, although could be difficult to set up in real-life settings [61]. The use of self-reports or pill counts could be alternative methods.

3. What is the reason and how to fix it? Given the numerous potential factors for non-adherence, it is therefore important to understand the reason(s) for non-adherence for each patient and to find an adequate personalized solution.

These three steps are in line with the Six Sigma framework to manage medication adherence developed by Vrijens et al. [62]. This framework includes definition (robust taxonomy), measure (operational definition and performance), analysis (understand the cause), addressing the cause and implementing solutions and measurement-guided adherence management.

In addition to the updated systematic review of adherence interventions in osteoporosis, a comprehensive list of interventions is provided with evidence about their effectiveness including systematic reviews of adherence interventions in other diseases including depression, cardiovascular diseases and hypercholesterolaemia [63-65] (Table 1). Patient education and counselling, adherence monitoring with feedback and dose simplification including flexible dosing regimen were associated with higher adherence improvements. We however acknowledge that the efficacy of these actions is still largely unknown and current data could be controversial.

Patient education and counselling were shown to improve post-fracture care and treatment initiation, with more controversial results on medication adherence. As a prominent example for post-fracture care, the PREVOST trial suggested that repeated oral and written information about fragility fractures and osteoporosis management by a case manager increased treatment initiation $(53 \%$ initiated post-fracture care in the intervention compared to $33 \%$ in the control) [66]. In another study [67], patient education and referral to endocrinologist by a nurse were shown to improve the initiation of calcium and vitamin D, although up to $50 \%$ of patients with osteoporosis did not complete follow-up visits and/or did not adhere to treatment recommendations for osteoporosis. Assigning a screening coordinator to identify, educate and follow up with fragility fracture patients and inform their physicians of the need to evaluate bone health was also shown to increase treatment initiation [68]. A systematic screening using FRAX ${ }^{\circledR}$ was also shown to increase use of, and adherence to anti-osteoporosis medications in the UK SCOOP Trial [69]. In addition, an osteoporosis school program (i.e. four classes of 8-12 participants over 4 weeks), peer-led community education and mentorship program or patient education program were associated with improved knowledge of osteoporosis and initiation of treatment [70]. In a large pragmatic randomized controlled trial, however, telephonic motivational interviewing intervention was not associated with significant improvements in medication adherence [59].

Adherence monitoring with feedback was also associated with improved adherence. Stuurman-Bieze et al. [71] showed that a proactive pharmaceutical care including counselling at baseline and at 2 weeks and an active monitoring and counselling every 3 months by pharmacists for patients who should have redeemed a new prescription leads to improved adherence. Ducomlombier et al. [72] further suggests that phone calls by medical secretaries every 2 months to motivate patients to maintain good adherence to the treatment and to detect any difficulties in adherence with the prescription using non-incriminating questions were associated with improved adherence.

Dose simplification including flexible dosing regimen was associated with improved adherence. Offering patients a medication with less strict administration instructions such as the use of gastro-resistant risedronate tablets that could be taken after breakfast was associated with improved persistence to treatment [73]. Longer dosing regimen (such as 6-month subcutaneous injection of denosumab or yearly intravenous injection of zoledronic acid) can also be interesting to improve adherence, although adherence levels have also been disappointed and far from optimal (see previously). Finally, a flexible dosing regimen (before breakfast; in-between meals; before bedtime) was also shown to be associated with improved 
Table 1 Evidence about the effectiveness of adherence-enhancing interventions

\begin{tabular}{llll}
\hline Intervention subclass & OP studies & Other conditions* & Conclusion \\
\hline Education and support & & & Mixed evidence of effectiveness \\
Patient education program & 4 & 4 & Strong evidence of effectiveness \\
Patient counselling & 1 & 10 & Strong evidence of effectiveness \\
Patient education combined with counselling & 11 & 15 & No evidence of effectiveness \\
Provision of educational material & 2 & 5 & Limited evidence of effectiveness \\
Monitoring supervision & 1 & Strong evidence of effectiveness \\
Non-adherence monitoring & 5 & 2 & Limited evidence of effectiveness \\
Adherence monitoring combined with counselling & 1 & 10 & Strong evidence of effectiveness \\
Drug regimen combined with counselling & 1 & 1 & No evidence of effectiveness \\
Reminders to take the medication & 3 & 7 & Strong evidence of effectiveness \\
Monitoring biomarkers & & $\mathrm{N} / \mathrm{a}$ & Limited evidence of effectiveness \\
Dose regimen adjustment and simplification & 4 & 8 & Limited evidence of effectiveness \\
Dose simplification including flexible dosing regimen & 1 & 1 & Mixed evidence of effectiveness \\
Individual medication program & $\mathrm{N} / \mathrm{a}$ & 1 & Low evidence of effectiveness \\
Costs covered & & 2 & 9
\end{tabular}

N/a not applicable

*Restricted to depression, cardiovascular diseases and hypercholesterolaemia

persistence, although there was no statistical difference in terms of adherence [74].

Improving patient interaction and shared decision-making can also lead to improve treatment initiation and potentially improve adherence. Several guidelines and international groups recommend that shared decision-making be part of standard treatment. In shared decision-making, both parties share information: the clinician offers options and describes their risks and benefits, and the patient expresses his or her preferences and values [75]. Each participant is thus armed with a better understanding of the relevant factors and shares responsibility in the decision about how to proceed [75]. Decision aids emphasize shared decision-making and include several features to support individualized treatment discussions like those needed for fracture prevention. Decision aids for a range of conditions have been shown to decrease decisional conflict, increase knowledge and (when probabilities are included in the aid) improve the accuracy of risk perception [76]. Some decision aids aiming to facilitate shared decisionmaking in osteoporosis have been designed and shown to improve the quality of clinical decisions about bisphosphonate therapy and may have improved adherence [77-79].

Providing patients with good quality information about osteoporosis, their risk and treatment options is needed as a crucial step to increase the diagnosis and treatment of osteoporosis. The doctor is therefore a key actor to improve medication adherence. As an educator and a partner in making treatment decisions, the doctor should be familiar with the medical evidence, able to discuss complex medical information in a manner that is understood by the patient and appropriately consider the patient's expectations, beliefs and concerns [80, 81]. A doctor should thus facilitate interaction with the patient and him/her, understand the patient's needs and investigate the type of treatment appropriate for the patients (e.g. dosing regimen).

\section{Recommendations for further research}

Some areas where further research is needed were identified. First, it is important to improve our understanding on what osteoporotic patients want, need and prefer and to better understand how and why patients will (not) be adherent. It is particularly important to understand the root causes for each aspect of adherence, i.e. initiation, implementation and persistence. Qualitative research is the best way to understand patients' needs and reasons for non-adherence and should be a starting point for designing appropriate interventions. Stated preference methods could also be interesting to reveal patients' preferences and important treatment characteristics [82].

Second, register-based studies can be attractive to investigate the importance of different factors and identify the strongest and most prevalent risk factors for poor adherence as targets for intervention. Big (pharmaco-epidemiologic) data provide new opportunities to help understand the reasons of medication non-adherence and may also prove helpful in assessing the effects of new initiatives in the area. 
Third, with a better understanding of patients' needs and non-adherence factors, it would then be possible to design interventions that fit patients' needs and wishes on a personalized manner. It is indeed recommended to develop complex, individualized and multifactorial interventions. There is not one single intervention to manage adherence. Involving patients in the preparation, development and assessment of these complex interventions would be crucial [83]. Further research is also warranted on patient involvement in medication adherence research and patient empowerment and its role in promoting adherence. Complex interventions would thus need to be tested in large scale randomized controlled trials.

Fourth, as the cost of an adherence intervention may be high, it would also be important to identify patients that will be receptive to interventions and spend our limited resources on those patients. We further need patient outcome tools to better understand the patient perspective and patient biases in decision-making concerning osteoporosis therapies [84].

In addition, web-based applications could play a crucial role in supporting patients to improve their adherence to medication. Further research focusing on digital adherence interventions is necessary to determine their value and ideal ways to improve osteoporosis medication adherence.

Other areas for further research include the need to adequately communicate about osteoporosis, fracture risk and osteoporosis treatment to patients; development of shared decision-making; assessment of adherence-management model (such as the three-stage model proposed previously); innovative methods for monitoring medication adherence; and policy/healthcare systems initiatives to support adherence interventions.

\section{Conclusions}

Osteoporosis represents a significant healthcare burden in European countries which, due to increasing life expectancies, is predicted to increase further in the future [24]. Despite the increasing burden and the availability of effective treatments in reducing the risk of fractures, most patients are not taking their medication appropriately or do not even start an osteoporosis medication. Improving treatment initiation and adherence to therapy is therefore urgently needed to leverage in full the benefits of drug therapy.

Poor and non-adherence with medication is common with a treatment gap estimated between and 95\% in European countries [25] and about half of the patients discontinuing therapy within 1 year. Reasons for non-adherence are numerous, diverse and multidimensional, depending on the interplay of multiple factors and may be different for each key element of adherence. Few single interventions have been shown to improve adherence or persistence to osteoporosis treatment. Potentially, promising interventions include patient education with counselling, adherence monitoring with feedback and dose simplification including flexible dosing regimen. Recommendations for practice and further research were provided, suggesting the need to emphasize the importance of adherence to treatment and to talk about adherence with patients, and the need to implement sound measures of adherence so that the intervention can be individualized. These recommendations are intended for clinicians to manage adherence of their patients and to researchers and policy makers to design, facilitate and appropriately use adherence interventions and to advance research in the field.

Acknowledgements The authors are grateful to the Prince Mutaib Chair for Biomarkers of Chronic Disease, King Saud University, Riyadh, KSA and the WHO Collaborating Centre for Public Health Aspects of Musculoskeletal Health and Aging, for its support.

Funding information The meeting was funded by the European Society for Clinical and Economic Aspects of Osteoporosis, Osteoarthritis and Musculoskeletal Diseases (ESCEO).

\section{Compliance with ethical standards}

Conflict of interest MLB has received honoraria from Amgen, Bruno Farmaceutici, Kyowa Kirin; academic grants and/or speaker from Abiogen, Alexion, Amgen, Bruno, Farmaceutici, Eli Lilly, Kyowa Kirin, MSD, NPS, Servier, Shire and SPA; and consulting fees from Alexion, Bruno Farmaceutici, Kyowa Kirin, Servier and Shire.

OB received grants research from Biophytis, IBSA, MEDA, Servier, SMB and consulting or lecture fees from Amgen, Biophytis, IBSA, MEDA, Servier, SMB, TRB Chemedica and UCB.

$\mathrm{BC}$ has received Fees for occasional interventions as an expert or speaker for Amgen, Expanscience, Ferring, Lilly, Medtronic, MSD, Mylan, Novartis, Roche Diagnostics and UCB.

$\mathrm{CC}$ has received lecture fees and honoraria from Amgen, Danone, Eli Lilly, GSK, Kyowa Kirin, Medtronic, Merck, Nestlé, Novartis, Pfizer, Roche, Servier, Shire, Takeda and UCB outside of the submitted work.

DC is funded by the Netherlands Organization for Health Research and Development (ZonMw), grant application number 848016001 .

ED has received honoraria from Pfizer and UCB.

ADP has received consulting and speaker fees from Amgen, UCB, Eli Lilly, Roche and Gilead and own shares of Active Life Sci.

JAK reports grants from Amgen, Eli Lilly and Radius Health and consulting fees from Theramex.

$\mathrm{PH}$ has received consulting and speaker fees from Amgen, Dr. Kade/Besin, Gedeon Richter, Elli Lilly, Meda/Mylan, Novartis, Pfizer, Roche, Theramex and UCB and has given expert testimony on behalf of Novartis.

RR has been speaker bureau or member of scientific advisory boards for Danone, Echolight, Effryx, Mylan, Nestlé, ObsEva, Pfizer, Radius Health, Sandoz and TEVA/Theramex.

TT reports personal fees for lectures and expertises from Abbvie, Amgen, Arrow, BMS, Chugai, Expanscience, Gilead, HAC-Pharma, LCA, Lilly, Medac, MSD, Pfizer, Theramex, Thuasne, TEVA and UCB and financial support or fees for research activities from Amgen, Bone Therapeutics, Chugai, HAC-Pharma, MSD, Novartis, Pfizer and UCB.

JYR has received consulting fees or paid advisory boards from IBSAGENEVRIER, Mylan, Radius Health, Pierre Fabre; lecture fees when speaking at the invitation of sponsor for IBSA-GENEVRIER, Mylan, CNIEL and Dairy Research Council; and grant support from industry (all through institution) from IBSA-GENEVRIER, Mylan, CNIEL and Radius Health. 
The other authors have no conflict of interest to declare relevant to the content of this study.

Open Access This article is distributed under the terms of the Creative Commons Attribution-NonCommercial 4.0 International License (http:// creativecommons.org/licenses/by-nc/4.0/), which permits any noncommercial use, distribution, and reproduction in any medium, provided you give appropriate credit to the original author(s) and the source, provide a link to the Creative Commons license, and indicate if changes were made.

\section{References}

1. Kanis JA, Cooper C, Rizzoli R, Reginster JY, Scientific Advisory Board of the European Society for C, Economic Aspects of O et al (2019) European guidance for the diagnosis and management of osteoporosis in postmenopausal women. Osteoporos Int 30(1):3-44

2. Hiligsmann M, McGowan B, Bennett K, Barry M, Reginster JY (2012) The clinical and economic burden of poor adherence and persistence with osteoporosis medications in Ireland. Value Health 15(5):604-612

3. Jha S, Wang Z, Laucis N, Bhattacharyya T (2015) Trends in media reports, oral bisphosphonate prescriptions, and hip fractures 19962012: an ecological analysis. J Bone Miner Res 30(12):2179-2187

4. Kanis JA, Cooper C, Rizzoli R, Abrahamsen B, Al-Daghri NM, Brandi ML et al (2017) Identification and management of patients at increased risk of osteoporotic fracture: outcomes of an ESCEO expert consensus meeting. Osteoporos Int 28(7):2023-2034

5. Sabate E (ed) (2003) Adherence to long-term therapies: evidence for action. World Health Organization, Geneva

6. Gold DT, Silverman S (2006) Review of adherence to medications for the treatment of osteoporosis. Curr Osteoporos Rep 4(1):21-27

7. Hiligsmann M, Salas M, Hughes DA, Manias E, Gwadry-Sridhar FH, Linck P, Cowell W (2013) Interventions to improve osteoporosis medication adherence and persistence: a systematic review and literature appraisal by the ISPOR Medication Adherence \& Persistence Special Interest Group. Osteoporos Int 24(12):29072918

8. Cornelissen D, et al. Interventions to improve osteoporosis medication adherence and persistence: an updated systematic review and literature appraisal In submission

9. Vrijens B, De Geest S, Hughes DA, Przemyslaw K, Demonceau J, Ruppar T et al (2012) A new taxonomy for describing and defining adherence to medications. Br J Clin Pharmacol 73(5):691-705

10. De Geest S, Zullig LL, Dunbar-Jacob J, Helmy R, Hughes DA, Wilson IB et al (2018) ESPACOMP Medication Adherence Reporting Guideline (EMERGE). Ann Intern Med 169(1):30-35

11. Lekkerkerker F, Kanis JA, Alsayed N, Bouvenot G, Burlet N, Cahall D, Chines A, Delmas P, Dreiser RL, Ethgen D, Hughes N, Kaufman JM, Korte S, Kreutz G, Laslop A, Mitlak B, Rabenda V, Rizzoli R, Santora A, Schimmer R, Tsouderos Y, Viethel P, Reginster JY, Group for the Respect of Ethics and Excellence in Science (GREES) (2007) Adherence to treatment of osteoporosis: a need for study. Osteoporos Int 18(10):1311-1317

12. Yeam CT, Chia S, Tan HCC, Kwan YH, Fong W, Seng JJB (2018) A systematic review of factors affecting medication adherence among patients with osteoporosis. Osteoporos Int 29(12):26232637

13. Fischer MA, Stedman MR, Lii J, Vogeli C, Shrank WH, Brookhart MA, Weissman JS (2010) Primary medication non-adherence: analysis of 195,930 electronic prescriptions. J Gen Intern Med 25(4): 284-290
14. Reynolds K, Muntner P, Cheetham TC, Harrison TN, Morisky DE, Silverman S, Gold DT, Vansomphone SS, Wei R, O'Malley CD (2013) Primary non-adherence to bisphosphonates in an integrated healthcare setting. Osteoporos Int 24(9):2509-2517

15. Rabenda V, Reginster JY (2010) Overcoming problems with adherence to osteoporosis medication. Expert Rev Pharmacoecon Outcomes Res 10(6):677-689

16. Rabenda V, Hiligsmann M, Reginster JY (2009) Poor adherence to oral bisphosphonate treatment and its consequences: a review of the evidence. Expert Opin Pharmacother 10(14):2303-2315

17. Strom O, Borgstrom F, Kanis JA, Compston J, Cooper C, McCloskey EV et al (2011) Osteoporosis: burden, health care provision and opportunities in the EU: a report prepared in collaboration with the International Osteoporosis Foundation (IOF) and the European Federation of Pharmaceutical Industry Associations (EFPIA). Arch Osteoporos 6:59-155

18. Landfeldt E, Strom O, Robbins S, Borgstrom F (2012) Adherence to treatment of primary osteoporosis and its association to fractures-the Swedish Adherence Register Analysis (SARA). Osteoporos Int 23(2):433-443

19. Blaschke TF, Osterberg L, Vrijens B, Urquhart J (2012) Adherence to medications: insights arising from studies on the unreliable link between prescribed and actual drug dosing histories. Annu Rev Pharmacol Toxicol 52:275-301

20. Rabenda V, Mertens R, Fabri V, Vanoverloop J, Sumkay F, Vannecke C, Deswaef A, Verpooten GA, Reginster JY (2008) Adherence to bisphosphonates therapy and hip fracture risk in osteoporotic women. Osteoporos Int 19(6):811-818

21. van Boven JFM, de Boer PT, Postma MJ, Vegter S (2013) Persistence with osteoporosis medication among newly-treated osteoporotic patients. J Bone Miner Metab 31(5):562-570

22. Roerholt C, Eiken P, Abrahamsen B (2009) Initiation of antiosteoporotic therapy in patients with recent fractures: a nationwide analysis of prescription rates and persistence. Osteoporos Int 20(2): 299-307

23. Jacob L, Dreher M, Kostev K, Hadji P (2016) Increased treatment persistence and its determinants in women with osteoporosis with prior fracture compared to those without fracture. Osteoporos Int 27(3):963-969

24. Svedbom A, Hernlund E, Ivergard M, Compston J, Cooper C, Stenmark J et al (2013) Osteoporosis in the European Union: a compendium of country-specific reports. Arch Osteoporos 8:137

25. Kanis JA, Svedbom A, Harvey N, McCloskey EV (2014) The osteoporosis treatment gap. J Bone Miner Res 29(9):1926-1928

26. Blaschke TF, Osterberg L, Vrijens B, Urquhart J. Adherence to medications: insights arising from studies on the unreliable link between prescribed and actual drug dosing histories. Annu Rev Pharmacol. 2012;52:275-+

27. Balasubramanian A, Brookhart MA, Goli V, Critchlow CW (2013) Discontinuation and reinitiation patterns of osteoporosis treatment among commercially insured postmenopausal women. Int J Gen Med 6:839-848

28. Wade SW, Satram-Hoang S, Nadkar A, Macarios D, Tosteson AN (2011) Impact of medication adherence on health care utilization and productivity: self-reported data from a cohort of postmenopausal women on osteoporosis therapy. Clin Ther 33(12):2006-2015

29. Siris ES, Selby PL, Saag KG, Borgstrom F, Herings RM, Silverman SL (2009) Impact of osteoporosis treatment adherence on fracture rates in North America and Europe. Am J Med 122(2 Suppl):S3$\mathrm{S} 13$

30. Ross S, Samuels E, Gairy K, Iqbal S, Badamgarav E, Siris E (2011) A meta-analysis of osteoporotic fracture risk with medication nonadherence. Value Health 14(4):571-581

31. Imaz I, Zegarra P, Gonzalez-Enriquez J, Rubio B, Alcazar R, Amate JM (2010) Poor bisphosphonate adherence for treatment of 
osteoporosis increases fracture risk: systematic review and metaanalysis. Osteoporos Int 21(11):1943-1951

32. Kyvernitakis I, Kostev K, Kurth A, Albert US, Hadji P (2014) Differences in persistency with teriparatide in patients with osteoporosis according to gender and health care provider. Osteoporos Int 25(12):2721-2728

33. Hadji P, Papaioannou N, Gielen E, Feudjo Tepie M, Zhang E, Frieling I, Geusens P, Makras P, Resch H, Möller G, KaloucheKhalil L, Fahrleitner-Pammer A (2015) Persistence, adherence, and medication-taking behavior in women with postmenopausal osteoporosis receiving denosumab in routine practice in Germany, Austria, Greece, and Belgium: 12-month results from a European non-interventional study. Osteoporos Int 26(10):2479-2489

34. Cheng LI, Durden E, Limone B, Radbill L, Juneau PL, Spangler L, Mirza FM, Stolshek BS (2015) Persistance and compliance with osteroporosis therapies among women in a commercially insured population in the United States. J Manag Care Spec Ph 21(9):824 U322

35. McClung MR, Wagman RB, Miller PD, Wang A, Lewiecki EM (2017) Observations following discontinuation of long-term denosumab therapy. Osteoporos Int 28(5):1723-1732

36. Lee YK, Nho JH, Ha YC, Koo KH (2012) Persistence with intravenous zoledronate in elderly patients with osteoporosis. Osteoporos Int 23(9):2329-2333

37. Ringe JD, Moller G (2009) Differences in persistence, safety and efficacy of generic and original branded once weekly bisphosphonates in patients with postmenopausal osteoporosis: 1year results of a retrospective patient chart review analysis. Rheumatol Int 30(2):213-221

38. Kanis JA, Reginster JY, Kaufman JM, Ringe JD, Adachi JD, Hiligsmann M, Rizzoli R, Cooper C (2012) A reappraisal of generic bisphosphonates in osteoporosis. Osteoporos Int 23(1):213-221

39. Hiligsmann M, Rabenda V, Gathon HJ, Ethgen O, Reginster JY (2010) Potential clinical and economic impact of nonadherence with osteoporosis medications. Calcif Tissue Int 86(3):202-210

40. Hiligsmann M, Rabenda V, Bruyere O, Reginster JY (2010) The clinical and economic burden of non-adherence with oral bisphosphonates in osteoporotic patients. Health Policy 96(2): 170-177

41. Danese MD, Badamgarav E, Bauer DC (2009) Effect of adherence on lifetime fractures in osteoporotic women treated with daily and weekly bisphosphonates. J Bone Miner Res 24(11):1819-1826

42. Strom O, Borgstrom F, Kanis J, Jonsson B (2009) Incorporating adherence into health economic modelling of osteoporosis. Osteoporos Int 20(1):23-34

43. Hiligsmann M, Boonen A, Rabenda V, Reginster JY (2012) The importance of integrating medication adherence into pharmacoeconomic analyses: the example of osteoporosis. Expert Rev Pharmacoecon Outcomes Res 12(2):159-166

44. Hiligsmann M, Reginster JY, Tosteson ANA, Bukata SV, Saag KG, Gold DT, Halbout P, Jiwa F, Lewiecki EM, Pinto D, Adachi JD, alDaghri N, Bruyère O, Chandran $M$, Cooper C, Harvey NC, Einhorn TA, Kanis JA, Kendler DL, Messina OD, Rizzoli R, Si L, Silverman S (2019) Recommendations for the conduct of economic evaluations in osteoporosis: outcomes of an experts' consensus meeting organized by the European Society for Clinical and Economic Aspects of Osteoporosis, Osteoarthritis and Musculoskeletal Diseases (ESCEO) and the US branch of the International Osteoporosis Foundation. Osteoporos Int 30(1):45-57

45. Landfeldt E, Strom O, Robbins S, Borgstrom F (2012) Adherence to treatment of primary osteoporosis and its association to fracturesthe Swedish Adherence Register Analysis (SARA). Osteoporos Int 23(2):433-443

46. Burkhart PV, Sabate E. Adherence to long-term therapies: evidence for action. J Nurs Scholarsh. 2003;35(3):207-
47. Kardas P, Lewek P, Matyjaszczyk M (2013) Determinants of patient adherence: a review of systematic reviews. Front Pharmacol 4

48. Johansson H, Siggeirsdottir K, Harvey NC, Oden A, Gudnason V, McCloskey $\mathrm{E}$ et al (2017) Imminent risk of fracture after fracture. Osteoporos Int 28(3):775-780

49. Beaton DE, Dyer S, Jiang D, Sujic R, Slater M, Sale JE et al (2014) Factors influencing the pharmacological management of osteoporosis after fragility fracture: results from the Ontario Osteoporosis Strategy's fracture clinic screening program. Osteoporos Int 25(1): 289-296

50. Majumdar SR, McAlister FA, Johnson JA, Weir DL, Bellerose D, Hanley DA et al (2014) Critical impact of patient knowledge and bone density testing on starting osteoporosis treatment after fragility fracture: secondary analyses from two controlled trials. Osteoporos Int 25(9):2173-2179

51. Morell S, Hemmeler C, Amsler F, Gross T (2017) Adherence to osteoporosis pharmacotherapy one year after osteoporotic fracture a Swiss trauma center secondary prevention project. Swiss Med Wkly 147:w14451

52. Yu J, Brenneman SK, Sazonov V, Modi A (2015) Reasons for not initiating osteoporosis therapy among a managed care population. Patient Prefer Adherence 9:821-830

53. Tafaro L, Nati G, Leoni E, Baldini R, Cattaruzza MS, Mei M, Falaschi P (2013) Adherence to anti-osteoporotic therapies: role and determinants of "spot therapy". Osteoporos Int 24(8):2319 2323

54. Iversen MD, Vora RR, Servi A, Solomon DH (2011) Factors affecting adherence to osteoporosis medications: a focus group approach examining viewpoints of patients and providers. J Geriatr Phys Ther 34(2):72-81

55. Papaioannou A, Kennedy CC, Dolovich L, Lau E, Adachi JD (2007) Patient adherence to osteoporosis medications: problems, consequences and management strategies. Drugs Aging 24(1):3755

56. Silverman SL, Schousboe JT, Gold DT (2011) Oral bisphosphonate compliance and persistence: a matter of choice? Osteoporos Int 22(1):21-26

57. Lau E, Papaioannou A, Dolovich L, Adachi J, Sawka AM, Burns S, Nair K, Pathak A (2008) Patients' adherence to osteoporosis therapy - exploring the perceptions of postmenopausal women. Can Fam Physician 54(3):394-402

58. Shu ADH, Stedman MR, Polinski JM, Jan SA, Patel M, Truppo C, Breiner L, Chen YY, Weiss TW, Solomon DH (2009) Adherence to osteoporosis medications after patient and physician brief education: post hoc analysis of a randomized controlled trial. Am J Manag Care 15(7):417-424

59. Solomon DH, Iversen MD, Avorn J, Gleeson T, Brookhart MA, Patrick AR, Rekedal L, Shrank WH, Lii J, Losina E, Katz JN (2012) Osteoporosis telephonic intervention to improve medication regimen adherence: a large, pragmatic, randomized controlled trial. Arch Intern Med 172(6):477-483

60. Delmas PD, Vrijens B, Eastell R, Roux C, Pols HA, Ringe JD et al (2007) Effect of monitoring bone turnover markers on persistence with risedronate treatment of postmenopausal osteoporosis. J Clin Endocrinol Metab 92(4):1296-1304

61. Vrijens B, Heidbuchel H (2015) Non-vitamin K antagonist oral anticoagulants: considerations on once- vs. twice-daily regimens and their potential impact on medication adherence. Europace 17(4):514-523

62. Vrijens B (2019) A Six Sigma framework to successfully manage medication adherence. Br J Clin Pharmacol 85:1661-1663

63. Rash JA, Campbell DJT, Tonelli M, Campbell TS (2016) A systematic review of interventions to improve adherence to statin medication: what do we know about what works? Prev Med 90:155169 
64. van Dalem J, Krass I, Aslani P (2012) Interventions promoting adherence to cardiovascular medicines. Int $\mathrm{J}$ Clin Pharm-Net 34(2):295-311

65. Chong WW, Aslani P, Chen TF (2011) Effectiveness of interventions to improve antidepressant medication adherence: a systematic review. Int J Clin Pract 65(9):954-975

66. Merle B, Chapurlat R, Vignot E, Thomas T, Haesebaert J, Schott AM (2017) Post-fracture care: do we need to educate patients rather than doctors? The PREVOST randomized controlled trial. Osteoporos Int 28(5):1549-1558

67. Seuffert P, Sagebien CA, McDonnell M, O'Hara DA. Evaluation of osteoporosis risk and initiation of a nurse practitioner intervention program in an orthopedic practice. Arch Osteoporos. 2016;11(1)

68. Beaton DE, Mamdani M, Zheng H, Jaglal S, Cadarette SM, Bogoch $\mathrm{ER}$, et al. Improvements in osteoporosis testing and care are found following the wide scale implementation of the Ontario Fracture Clinic Screening Program: an interrupted time series analysis. Medicine. 2017;96(48)

69. Parsons C, Harvey N, Shepstone L, Kanis J, Lenaghan E, Clarke S, et al. Systematic screening using Frax leads to increased use of, and adherence to, anti-osteoporosis medications: the Uk Scoop Trial. Osteoporosis Int. 2018;29:613-

70. Nielsen D, Ryg J, Nielsen W, Knold B, Nissen N, Brixen K (2010) Patient education in groups increases knowledge of osteoporosis and adherence to treatment: a two-year randomized controlled trial. Patient Educ Couns 81(2):155-160

71. Stuurman-Bieze AGG, Hiddink EG, van Boven JFM, Vegter S (2014) Proactive pharmaceutical care interventions decrease patients' nonadherence to osteoporosis medication. Osteoporos Int 25(6):1807-1812

72. Ducoulombier V, Luraschi H, Forzy G, Vandecandelaere M, Houvenagel E (2015) Contribution of phone follow-up to improved adherence to oral osteoporosis treatment. Am J Pharm Benefist 7(3):E81-EE9

73. Hiligsmann M, Reginster JY (2019) Cost-effectiveness of gastroresistant risedronate tablets for the treatment of postmenopausal women with osteoporosis in France. Osteoporos Int 30(3):649-658

74. Oral A, Lorenc R, Investigators F-AS (2015) Compliance, persistence, and preference outcomes of postmenopausal osteoporotic women receiving a flexible or fixed regimen of daily risedronate: a multicenter, prospective, parallel group study. Acta Orthop Traumatol Turc 49(1):67-74
75. Barry MJ, Edgman-Levitan S (2012) Shared decision makingpinnacle of patient-centered care. N Engl J Med 366(9):780-781

76. Stacey D, Legare F, Lewis K, Barry MJ, Bennett CL, Eden KB et al (2017) Decision aids for people facing health treatment or screening decisions. Cochrane Db Syst Rev 4

77. Montori VM, Shah ND, Pencille LJ, Branda ME, Van Houten HK, Swiglo BA et al (2011) Use of a decision aid to improve treatment decisions in osteoporosis: the osteoporosis choice randomized trial. Am J Med 124(6):549-556

78. Hiligsmann M, Ronda G, van der Weijden T, Boonen A (2016) The development of a personalized patient education tool for decision making for postmenopausal women with osteoporosis. Osteoporos Int 27(8):2489-2496

79. Smallwood AJ, Schapira MM, Fedders M, Neuner JM (2017) A pilot randomized controlled trial of a decision aid with tailored fracture risk tool delivered via a patient portal. Osteoporos Int 28(2):567-576

80. Lewiecki EM (2010) Risk communication and shared decision making in the care of patients with osteoporosis. J Clin Densitom 13(4):335-345

81. Gasparik AI (2014) Clinical setting influences patterns of interaction between osteoporosis patient and physician. Calcif Tissue Int 95(2):122-124

82. Hiligsmann M, Pinto D, Dennison E, Al-Daghri N, Beaudart C, Branco J et al (2019) Patients' preferences for osteoarthritis treatment: the value of stated-preference studies. Aging Clin Exp Res 31(1):1-3

83. de Wit M, Cooper C, Tugwell P, Bere N, Kirwan J, Conaghan PG et al (2019) Practical guidance for engaging patients in health research, treatment guidelines and regulatory processes: results of an expert group meeting organized by the World Health Organization (WHO) and the European Society for Clinical and Economic Aspects of Osteoporosis, Osteoarthritis and Musculoskeletal Diseases (ESCEO). Aging Clin Exp Res 31(7):905-915

84. Silverman S, Gold DT (2018) Medication decision-making in osteoporosis: can we explain why patients do not take their osteoporosis medications? Current Osteoporosis Reports 16(6):772-774

Publisher's note Springer Nature remains neutral with regard to jurisdictional claims in published maps and institutional affiliations.

\section{Affiliations}

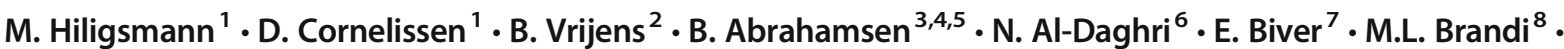

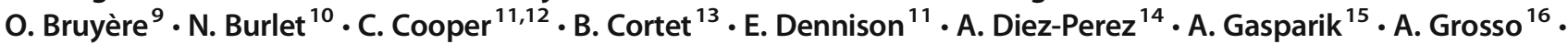
P. Hadji ${ }^{17}$ • P. Halbout ${ }^{18}$ • J.A. Kanis ${ }^{19,20}$ - J.M. Kaufman ${ }^{21}$ - A. Laslop ${ }^{22} \cdot$ S. Maggi ${ }^{23} \cdot$ R. Rizzoli ${ }^{7}$. T. Thomas $^{24}$. S. Tuzun ${ }^{25} \cdot$ M. Vlaskovska ${ }^{26} \cdot$ J.Y. Reginster ${ }^{6,9}$

1 Department of Health Services Research, CAPHRI Care and Public Health Research Institute, Maastricht University, P.O. Box 616, 6200 MD Maastricht, the Netherlands

2 Research and Development, AARDEX Group and Department of Public Health, University of Liège, Liege, Belgium

3 Open Patient Data Explorative Network, Institute of Clinical Resesarch, University of Southern Denmark, Odense, Denmark

4 Department of Medicine, Holbæk Hospital, Holbæk, Denmark

5 NDORMS, University of Oxford, Oxford, UK
6 Chair for Biomarkers of Chronic Diseases, Biochemistry Department, College of Science, King Saud University, Riyadh, Saudi Arabia

7 Division of Bone Diseases, Geneva University Hospitals and Faculty of Medicine, Geneva, Switzerland

8 FirmoLab, Fondazione FIRMO e Università di Firenze, Florence, Italy

9 Division of Public Health, Epidemiology and Health Economics, Liège, Belgium and WHO Collaborating Centre for Public Health 
Aspects of Musculoskeletal Health and Aging, University of Liège, Liege, Belgium

10 Global Head of Patient Insights Innovation, Patient Solution Unit, Sanofi, Lyon, France

11 MRC Lifecourse Epidemiology Unit, Southampton General Hospital, University of Southampton, Southampton, UK

12 NIHR Musculoskeletal Biomedical Research Unit, University of Oxford, Oxford, UK

13 Department of Rheumatology and EA 4490, University-Hospital of Lille, Lille, France

14 Musculoskeletal Research Unit, IMIM-Parc Salut Mar, CIBERFES, Universitat Autònoma de Barcelona, Barcelona, Spain

15 Department of Public Health, University of Medicine, Pharmacy, Science and Technology of Targu Mures, Targu Mures, Romania

16 Patient partner, Geneva, Switzerland

17 Frankfurt Centre of Bone Health, Frankfurt, Germany \& PhilipsUniversity of Marburg, Marburg, Germany
18

19

Centre for Metabolic Bone Diseases, University of Sheffield Medical School, Sheffield, UK

20 Mary McKillop Health Institute, Catholic University of Australia, Melbourne, Australia

21 Department of Endocrinology, Ghent University Hospital, Ghent, Belgium

22 Scientific Office, Austrian Federal Office for Safety in Health Care, Vienna, Austria

23 CNR Aging Branch-NI, Padua, Italy

24 Department of Rheumatology, Hôpital Nord, CHU Saint-Etienne, Saint-Etienne and INSERM U1059, Université de Lyon-Université Jean Monnet, Saint-Etienne, France

25 Department of Physical Medicine and Rehabilitation, Cerrahpaşa Medical Faculty, İstanbul University Cerrahpaşa, Istanbul, Turkey

26 Medical Faculty, Department of Pharmacology, Medical University Sofia, Sofia, Bulgaria 